Irish Math. Soc. Bulletin

Number 70, Winter 2012, 37-38

ISSN 0791-5578

\title{
SYMBOL ALGEBRAS, INVOLUTIONS AND TRACE FORMS
}

\author{
RONAN FLATLEY
}

This is an abstract of the $\mathrm{PhD}$ thesis Symbol algebras, involutions and trace forms written by Ronan Flatley under the supervision of Doctor Thomas Unger and Professor David W. Lewis at the School of Mathematical Sciences, University College Dublin and submitted in August 2011.

Let $n$ be an arbitrary positive integer and let $K$ be a field of characteristic different from 2 containing a primitive $n^{\text {th }}$ root of unity $\omega$. Let $a, b \in K^{\times}$and $S$ be the algebra over $K$ generated by elements $x$ and $y$ subject to the relations $x^{n}=a, y^{n}=b$ and $y x=\omega x y$. We call $S$ a symbol algebra. Symbol algebras are central simple algebras and are generalisations of quaternion algebras. We construct examples of symbol algebras of arbitrary degree.

Let $\operatorname{Trd}_{S}$ be the reduced trace of $S$. The trace form $T_{S}:\left(s_{1}, s_{2}\right) \mapsto$ $\operatorname{Trd}_{S}\left(s_{1} s_{2}\right)$ is a symmetric bilinear form on $S$ with values in $K$. Given an arbitrary symbol algebra $S$, we compute its trace form $T_{S}$. We obtain distinct formulae for algebras of even and odd degree. In the even degree case we establish some trace form criteria to determine if the algebra $S$ is a division algebra. We also compute exterior powers of trace forms of symbol algebras.

We investigate symbol algebras with involution $(S, \sigma)$ where the involution $\sigma$ is of the second kind, which means that the centre of the algebra $S$ is a quadratic extension of the subfield $F$ of $K$, fixed by $\left.\sigma\right|_{K}$. The involution trace form $T_{(S, \sigma)}:\left(s_{1}, s_{2}\right) \mapsto \operatorname{Trd}_{S}\left(\sigma\left(s_{1}\right) s_{2}\right)$ is a $K / F$-hermitian form. We investigate certain natural classes of involutions of the second kind on symbol algebras and compute their corresponding involution trace forms. Such involutions are representatives of their class of involutions which restrict to conjugation on

2010 Mathematics Subject Classification. 11E81, 16K20.

Key words and phrases. symbol algebra, trace form, exterior power, involution.

Received on 20-11-2012.

Support from Science Foundation Ireland through the Research Frontiers Programme (project number 07/RFP/MATF191) is gratefully acknowledged. 
$K$. We give relations between the coefficients of certain invertible elements of $S$ which generate other involutions within their respective classes. We also examine the signatures of certain involution trace forms over formally real fields.

Department of Mathematics and Computer Studies, Mary ImmaCulate College, Limerick, Ireland

E-mail address: ronan.flatley@mic.ul.ie 